The frequency of ECT remains largely a matter of tradition, with little consistency in the literature. We (Gangadhar et al, 1993) have also found that twiceweekly bilateral ECT was comparable to a thriceweekly schedule for melancholic depressive patients, half of whom were drug-naïve. The depression scores reduced comparably in a given timeframe, despite the twice-weekly ECT group receiving significantly fewer ECTs. ECTs given twice a week had the advantage of obtaining optimal seizure duration in all ECT sessions (Janakiramaiah et al, 1992).

The matter is somewhat unsettled once again by Kellner et al (1992). They demonstrated a more rapid antidepressant effect with bilateral ECT, three times a week, than with once-a-week bilateral ECT in geriatric depressives.

Until the heterogeneity of patient characteristics, type of depression and prior medication are taken into account, studies of the onset as well as rate of improvement with ECT cannot be expected to yield consistent conclusions. The suggestion by Drs Scott \& Whalley to examine different schedules of ECT in a large population of depressives is hence especially timely. So also is the need to incorporate more sensitive techniques to monitor even smaller, early changes that may be induced by ECT in depression.

Gangaibhar, B. N., Janakiramaiah, N., Subbakrishna, D. K., et al (1993) Twice versus thrice weekly ECT in melancholia: a double blind prospective comparison. Journal of Affective Disorders, 27, 273-278.

Jagiadersh, H. N., Gangadhar, B. N., Janakiramaiah, N., et al (1992) Time dependent therapeutic effect of single electroconvulsive therapy (ECT) in endogenous depression. Journal of Affective Disorders. 24, $291-296$.

Janakiramaiah, N., Jyoti RaO, K. M.. Praveien, J., el al (1992) Seizure duration over ECT sessions: influence of spacing ECTs. Indian Journal of Psychiatry, 34, 124 127

Kellaner. C. H., Monrot, R. R., Pritchett, J.. el al (1992) Weekly ECT in geriatric depression. Convulsive Therapy, 8, 245253.

B. N. Gangadhar

N. JANAKIRAMAIAH

National Institute of

Mental Health and Neuro Sciences

Bangalore-560029, India

\section{Thalamo-frontal psychosis}

SIR: The suggested association by McGilchrist et al (BJP, July 1993, 163, 113-115) between thalamic lesions and a cyclical psychosis is intriguing. The authors correctly assert that theirs is the first clear report of cyclical psychosis following thalamic infarction. A careful reading of the literature may allow further insights into the possible significance of the case.
A total of 34 cases (McGilchrist et al, 1993; Starkstein et al, 1988a; Trimble \& Cummings, BJP, January $1981,138,56-59)$ of brain injury leading to mania have been reported. Fifteen of these cases had diencephalic lesions, eight frontal lobe, seven temporal lobe and the remaining four had widespread lesions. Twenty-one of the 34 had right-sided lesions, ten bilateral and three had isolated left-sided lesions. Six cases had evidence of damage in the thalamus, five of which were right and one bilateral (McGilchrist et al, 1993); none was left-sided. Two other cases had right thalamic and brainstem lesions.

The second of Trimble \& Cummings' two cases had a cyclical psychosis and a right thalamic arteriovenous malfunction which had bled into the ventricles (in addition, a lucency in the upper brainstem had been noted and at the time more significance was attributed to this aspect of the case). Six to 12 months after the intraventricular haemorrhage, this patient developed a manic-depressive-like illness which deteriorated gradually over the next five years into a schizophreniform state. Almost all the cases have lesions of the frontal lobe or its connections (Starkstein et al, 1988a). In addition, most of the lesions are right-sided.

Starkstein et al (1988b) addressed the specific role of basal ganglia and thalamus in different mood changes. They found a strong association between basal ganglia (more commonly the left and more specifically the caudate head) and post-stroke depression in contrast to the thalamus. They explain this finding on the basis of the anatomy of the biogenic amine pathways. The biogenic amine pathways run through the basal ganglia but are ventral to the thalamus. Starkstein et al have thus argued that they are spared in thalamic lesions and involved in basal ganglionic lesions. However, this hypothesis does not explain the association of euphoria and mania secondary to thalamic damage.

As we have pointed out earlier, it is a right-sided thalamic lesion which is associated with secondary mania. Oke et al (1978) have demonstrated lateralisation of norepinephrine (NE) in the human thalamus. The ventral tier, centromedian and parafascicular nuclei of the right thalamus contain much greater NE than do those areas on the left (Oke et al, 1978). Starkstein et al $(1988 a)$ have proposed that the induction of receptor supersensitivity by mesocorticolimbic dopaminergic or NE pathway lesions, or both, may mediate the neurobehavioural expression of mania. It is thus possible to explain the association of mania and right thalamic lesions on the basis of laterality of NE distribution in the thalamus.

Of course, the current state of knowledge does not permit a comprehensive explanation of mood 
changes associated with particular lesion lateralisation or location. However, any hypothesis of the mechanisms of mood disturbance following focal brain injury must account for the apparent association of left anterior lesions with depression and of right-sided lesions with euphoria. It is to be hoped that as our understanding of the subcortical substrates of behaviour increases, we will be able to better understand the pathogenesis of cases such as that reported (McGilchrist et al, 1993).

OKe, A., Keller, R., Mefrord, I., et al (1978) Lateralisation on norepinephrine in human thalamus. Science, 200, 1411-1413.

Starkstein, S. E., Boston, J. D. \& Robinson, R. G. (1988a) Mechanisms of mania after brain injury: 12 case reports and review of the literature. Journal of Nervous and Mental Disease, 176, 87-100.

-, Robinson, R. G., BerthiER, M. L., et al (1988b) Differential mood changes following basal ganglia vs thalamic lesions. Archives of Neurology, 45, 725-730.

ANOOP RANJAN VARMa JOHN MORIARTY

MichaEL, R. TRIMBLE

Raymond Way Neuropsychiatry Research Group

Institute of Neurology

Queen Square

London WCIN $3 B G$

\section{Parental bonding}

SIR: Morris' letter (BJP, July 1993, 163, 127) criticising my paper (BJP, March 1993, 162, 335-344) requires a reply. Bowlby's definition of 'a poor early relationship' in the passage I cited was too general to be incorporated into a testable hypothesis. Since Bowlby went on to refer to the individual's "capacity to make affectional bonds", it seemed that the most precise hypothesis I could test was that there was a relationship between recalling having good affectional bonds in childhood and the ability to make good affectional bonds in adult life. Maternal care was the experience which came closest to having good affectional bonds. In the two samples I used, this was assessed by interview and, in the younger sample, it was also assessed by the care scale of the mother version of Parker's Parental Bonding Instrument (PBI). In an earlier paper (BJP, December 1988, 153, 758-769) I reported upon findings using scales of both the mother and the father versions of the PBI. Focusing specifically upon the care scale is a perfectly legitimate exercise. To be consistent, I also focused specifically upon the affection-given scale of the Marital Patterns Test.

It seems that what Morris is suggesting is that I should extend the study to testing the additional hypothesis that being subjected to parental over- protection in childhood (the other scale of the PBI) predisposes the individual to either seek or avoid overprotection in later relationships, and that I should use the directiveness scale of the self-rating and partner-rating questionnaires (BJP, May 1991, $158,648-657)$ as a measure of such overprotection. This is indeed a reasonable suggestion since Parker quite often (and more correctly I think) refers to the overprotection scale as the control scale, and control and directiveness are comparable constructs. Since, however, in the 1988 paper there was shown to be a highly significant association between recalled maternal overprotection and depression, the intervening variable of depression is going to complicate matters as it did with the maternal care hypothesisan issue which Morris studiously avoids.

Morris refers to "the total parental relationship" as getting closer to Bowlby's hypothesis. In the passage I cited, Bowlby used the term "an individual's experiences with his parents". This is a vague term which can hardly be preferable to defining the recollected relating behaviour of one or other parent in certain specific ways. I end my paper by maintaining that further progress in this field will depend upon expanding the theoretical base, increasing the range of parameters of relating to be considered, and developing new instruments which are based upon this broader perspective. In my recent book (Birtchnell, 1993) this is what I have tried to do.

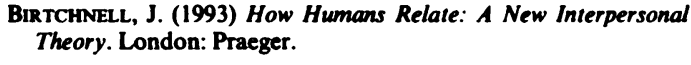

\section{Neural network model of amnesia}

SIR: Neural network modelling is a rapidly advancing area which, if appropriately applied, may be useful in psychiatry. The article by Carrie (BJP, August 1993, $163,217-222)$ is therefore welcomed with enthusiasm. Several comments are, however, important.

A simple associative network is used and the author chooses to represent diffuse cerebral pathology by random deletion of units. It is important to realise that units are substrates for the representation of patterns (during presentation and retrieval), and the memories for the patterns are stored in the synaptic weights between the units (which change before and after learning). Removal of units will naturally affect 'retrieval' due to the lack of a proper platform for the 